\title{
QUALITATIVE ANALYSIS OF ROTATIONAL MODES WITHIN THREE DIMENSIONAL EMPIRICAL MODE DECOMPOSITION
}

\author{
Naveed ur Rehman and Danilo P. Mandic \\ Imperial College London, UK. \\ Email: \{naveed.rehman07, d.mandic\}@ic.ac.uk
}

\begin{abstract}
An analysis of quaternion-valued intrinsic mode functions (IMFs) within three dimensional empirical mode decomposition is presented. This is achieved by using the delay vector variance (DVV) method, which examines the signal predictability in phase space to assess the determinism and nonlinearity within the signal. The study illustrates that the contribution of the first few IMFs contain information related to the stochastic/nonlinear signal nature, whereas the lower order IMFs are largely deterministic. The analysis is supported by simulation results on a quaternion signal composed of linear/nonlinear benchmark signals and on real world wind data.
\end{abstract}

Index Terms- Quaternion signal processing, Empirical mode decomposition (EMD), Intrinsic mode functions (IMFs), Delay vector variance (DVV).

\section{INTRODUCTION}

The empirical mode decomposition (EMD) algorithm, designed for time frequency analysis of nonlinear and nonstationary data, decomposes the signal in hand into a number of oscillatory modes, called intrinsic mode functions (IMFs) [1]. These intrinsic mode functions are 'well behaved' in terms of localized time-frequency representation; so that the subsequent application of Hilbert transform yields meaningful time-frequency-amplitude spectrum [1].

Recently, extensions of EMD to higher dimensions have been proposed in order to enable processing of multivariate (bivariate and pure quaternion) signals directly in the domain where they reside. These extensions include complex EMD [2], rotation-invariant complex EMD [3], and bivariate EMD [4] for complex/bivarite signals, whereas a three dimensional empirical mode decomposition algorithm has been recently proposed by Rehman and Mandic [5] to process pure quaternion (trivariate) signals. These extensions are not trivial, as e.g. finding the extrema of a complex signal is rather challenging, since $\mathbb{C}$ is not an ordered field. The three dimensional EMD algorithm is generic in a sense that, for complex (bivariate) signals and the proper choice of axis of rotation, it is isomorphic to the rotation invariant and bivariate EMD algorithms. It yields pure quaternion (real part is zero) IMFs, which carry physical meaning of rotation in 3D spaces, in the same way the real-valued IMFs, extracted from standard EMD, represents oscillatory modes within the signal. However, in order to make full use of three dimensional EMD, several key issues, such as local orthogonality of the quaternion-valued IMFs are still to be understood. An insight in to the role of quaternion-valued IMFs is therefore a prerequisite to applying the algorithm to real world nonlinear and nonstationary signals.

Signal modality characterisation, that is, the degree of linearity and nonlinearity, and determinism and stochasticity, within a signal is of great interest in modern signal processing [6]; for instance, the change in the nature of heart signals from stochastic to deterministic may indicate health hazard. Since EMD is being increasingly applied in a variety of applications where signals are of nonlinear and nonstationary nature [7], it is crucial to establish whether and how the modality of the original signal is altered by EMD. To this end, Chen $e t$ $a l$. have provided a qualitative assessment of the IMFs of the standard real valued EMD [8].

In this paper, we set out to investigate some intricate properties of the quaternion (pure) IMFs, generated by three dimensional EMD, to establish whether they preserve the nature of the original signal in phase space. This is important, for instance, in 'data fusion via fission' for the modelling of the relevance of input variables [9]. The delay vector variance (DVV) method is used for this purpose, as it is based on the predictability in phase space, and quantifies the degree of determinism and nonlinearity within the signal [6]. Since three dimensional EMD caters for both the complex and quaternion signals, the analysis is valid for both the complex and pure quaternion signals.

\section{THREE DIMENSIONAL EMD}

The three dimensional EMD method has been proposed recently to process trivariate (pure quaternion) signals directly in the quaternion domain [5]. In order to obtain the local mean, a critical step in the sifting algorithm, the algorithm employs unit quaternions for rotation in 3D spaces, thus providing projections of multidimensional signal in several directions. These projections are then used to calculate the enve- 


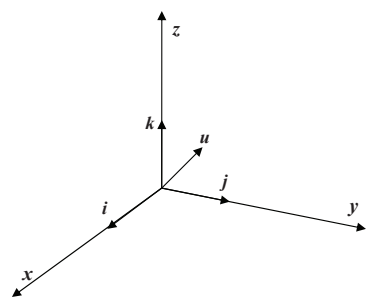

(a)

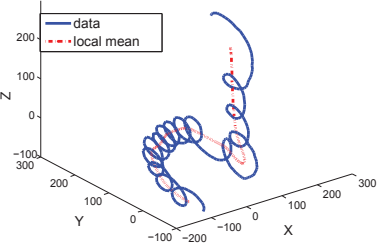

(b)
Fig. 1. Three Dimensional EMD Method: (a) Choices for axes of rotation: The axes of rotation can be selected as unit vectors along $x, y$, and $z$ axes, or as a 3D unit vector, $\mathbf{u}=\frac{(\mathbf{i}+\mathbf{j}+\mathbf{k})}{\sqrt{3}}=\frac{\imath+\jmath+\kappa}{\sqrt{3}}$; (b) Local mean calculation for a three dimensional signal using $\mathrm{K}=32$.

lope curves by interpolating their maxima using componentwise spline interpolation. These envelopes are then averaged to obtain the local mean of an input 3D signal.

A major difference between rotations in 3D spaces and $2 \mathrm{D}$ planes is the specification of the axis of rotation, which defines the direction about which a vector is rotated by a given angle. The rotation axis can be chosen as one of the three unit vectors, represented by $\imath, \jmath$, and $\kappa$ in Figure 1(a). If $\imath$ is chosen to be the axis of rotation, and the input signal is represented by $v(t)=a(t) \imath+b(t) \jmath+c(t) \kappa$, then in the quaternion domain, the rotation of $v(t)$ by an angle $2 \theta$ is given by

$$
\begin{aligned}
p_{\theta}^{\imath}= & e^{\imath \theta} v(t)\left(e^{\imath \theta}\right)^{*} \\
= & a \imath+(b \cos 2 \theta-c \sin 2 \theta) \jmath+ \\
& (c \cos 2 \theta+b \sin 2 \theta) \kappa
\end{aligned}
$$

where $p_{\theta}^{\imath}$ is the projection of $v(t)$ in the direction of $\theta$. Similarly, if $\jmath$ and $\kappa$ are selected as axes of rotations, then the rotated signals will have a form similar to that of (1). Observe from (1), that for projections along $\jmath$ and $\kappa$, only two of the three directions are being used to calculate the local mean of a 3D signal, which results in approximate estimates.

To circumvent this problem, a 3D unit vector $\mathbf{u}$ is selected as an axis of rotation, which has components in all the directions, that is, $\mathbf{u}=\frac{(\imath+\jmath+\kappa)}{\sqrt{3}}$. In this case, the projection of a signal rotated by an angle $2 \theta$ about unit vector $\mathbf{u}$ along the $\imath$ axis, can be calculated as

$$
\begin{aligned}
p_{\theta}^{\mathbf{u}}= & \Re\left[e^{\mathbf{u} \theta} v(t)\left(e^{\mathbf{u} \theta}\right)^{*} \cdot \imath\right] \\
= & \Re\left[e^{\left(\frac{\imath+++\kappa}{\sqrt{3}}\right) \theta} v(t)\left(e^{\left(\frac{-\imath-\jmath-\kappa}{\sqrt{3}}\right) \theta}\right) \cdot \imath\right] \\
= & a \cos ^{2} \theta+\frac{1}{3}(2 b+2 c-a) \sin ^{2} \theta+ \\
& \frac{1}{\sqrt{3}}(c-b) \sin 2 \theta
\end{aligned}
$$

where "." denotes the dot product and $\Re(\cdot)$ extracts the real component of the projection.
Observe from (2) that the projected signal contains all the three dimensions of $v(t)$, and therefore an accurate estimate of the local mean is obtained. In order to calculate the envelopes in multiple directions, angle $\theta$ can be selected to have $K$ values between 0 to $\pi$, that is, $\theta_{k}=(k \pi) / K$, where $1 \leq$ $k \leq K$. Notice the upper bound of $\pi$, since unit quaternions provide rotation by an angle $2 \theta$. If we choose $K=6$, and use $\mathbf{u}=\frac{(\imath+\jmath+\kappa)}{\sqrt{3}}$ as an axis of rotation, then from (2), the extrema along all the three components of the signal are employed to obtain the projections. Figure 1(b) illustrates the performance of the proposed method for the calculation of a local mean of a quaternion signal, using $K=32$.

Let $v(t)$ be a trivariate signal represented by a pure quaternion. For an initial value of $k=1$, the 3D extension of EMD can be summarized as

1. Calculate the projection of $v(t), p_{\theta_{k}}^{\mathbf{u}}$, in the direction $\theta_{k}$, along an axis of rotation $\mathbf{u}$, using (2);

2. Find the time instants $\left\{t_{k}^{\mathbf{u}}\right\}$ corresponding to the maxima of $p_{\theta_{k}}^{\mathbf{u}}(t)$;

3. Interpolate $\left[t_{k}^{\mathbf{u}}, v\left(t_{k}^{\mathbf{u}}\right)\right]$ to obtain envelope curves $e_{\theta_{k}}^{\mathbf{u}}$;

4. If $k<K$, then set $k=k+1$ and go to step 1, else continue;

5. Compute the mean $m(t)$ of all envelope curves $\left\{e_{\theta_{k}}^{\mathbf{u}}\right\}_{k=1}^{K}$;

6. Extract the "detail" $d(t)$ using $d(t)=v(t)-m(t)$.

If the "detail" $d(t)$ fulfills the stoppage criteria of IMF, then it is selected as an IMF and the above procedure is applied to $v(t)-d(t)$, otherwise it is applied to $d(t)$. This process is continued until all the rotational modes within the signal are obtained.

\section{DELAY VECTOR VARIANCE (DVV)}

The Delay vector variance (DVV) method [6] uses predictability of the signal in phase space to examine the determinism and nonlinearity within a signal. If $m$ represents an optimal embedding parameter, then DVV method can be summarized as follows:

1. The mean, $\mu_{d}$, and standard deviation, $\sigma_{d}$, are computed over all pairwise euclidean distances between delay vectors (DVs), $\|\mathbf{x}(i)-\mathbf{x}(j)\|(i \neq j)$;

2. The sets of 'neighbouring' delay vectors $\Omega_{k}\left(r_{d}\right)$ are generated, such that, $\Omega_{k}\left(r_{d}\right)=\{\mathbf{x}(i) \mid\|\mathbf{x}(k)-\mathbf{x}(i)\| \leq$ $\left.r_{d}\right\}$, that is, sets which consists of all DVs that lie closer to $\mathbf{x}(k)$ than a certain distance $r_{d}$, taken from the interval $\left[\max \left\{0, \mu_{d}-n_{d} \sigma_{d}\right\} ; \mu_{d}+n_{d} \sigma_{d}\right]$, where $n_{d}$ is a parameter controlling the span over which to perform the DVV analysis for $N_{t v}$ uniformly spaced distances; 
3. For every set $\Omega_{k}\left(r_{d}\right)$, the variance of the corresponding targets, $\sigma_{k}^{2}\left(r_{d}\right)$ is computed. The average over all sets $\Omega_{k}\left(r_{d}\right)$ normalised by the variance of the time series, $\sigma_{x}^{2}$, yields the target variance $\sigma^{* 2}\left(r_{d}\right)$,

$$
\sigma^{* 2}\left(r_{d}\right)=\frac{\frac{1}{N} \sum_{k=1}^{N} \sigma_{k}^{2}\left(r_{d}\right)}{\sigma_{x}^{2}}
$$

Variance measurements from (3) are considered valid only if the corresponding set $\Omega_{k}\left(r_{d}\right)$ contains atleast $N_{o}=30 \mathrm{DVs}$, to avoid unreliable estimates of variance.

The target variance is plotted as a function of standardised distance $\left(\frac{r_{d}-\mu_{d}}{\sigma_{d}}\right)$ to get DVV plots in which the presence of strong deterministic component yields small target variances $\sigma^{* 2}\left(r_{d}\right)$ for small spans $r_{d}$. To check for the nonlinearity within a signal, averaged DVV plots over a number of surrogate signals (generated using the hypothesis of linearity) can be generated and conveniently combined in a scatter diagram, where the horizontal axis corresponds to the target variance of the original time series and the vertical to that of the surrogate time series.

\section{HOW INFORMATIVE ARE QUATERNION IMF'S}

To provide qualitative assessment of the quaternion-valued IMFs generated from three dimensional EMD, following on [8], we shall employ the DVV method. The DVV scatter plots are generated for two cases: the sum of first and second quaternion IMFs, and the sum of all the remaining IMFs. The root mean squared (rms) value is calculated over the difference between the local variance of the original signal and that of individual IMFs. This metric is then used to quantify the degree of similarity between the DVV scatter plots of the original signal and that of the IMFs, and is given by

$$
\epsilon=\sqrt{\frac{1}{M} \sum_{\text {valid } r_{d}}\left(\sigma_{\text {ori }}^{* 2}\left(r_{d}\right)-\sigma_{\text {imf }}^{* 2}\left(r_{d}\right)\right)^{2}},
$$

where $\sigma_{\text {ori }}^{* 2}\left(r_{d}\right)$ denotes target variance, at span $r_{d}$, for the original signal, whereas $\sigma_{i m f}^{* 2}\left(r_{d}\right)$ denotes the variance value for an individual IMF, and $M$ is the number of valid variance $\left(r_{d}\right)$ values. The greater the value of the above metric for any two given signals, the greater the difference in their fundamental properties in phase space.

To perform both the qualitative and quantitative analysis of IMFs, for illustration, we generated a pure quaternion signal, consisting of three signals of different nature, that is

$$
Q=0+X \imath+Y \jmath+Z \kappa
$$

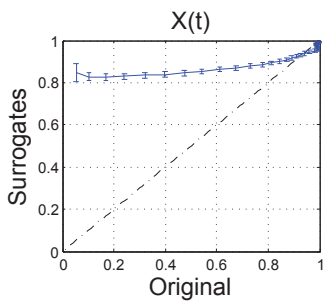

(a)

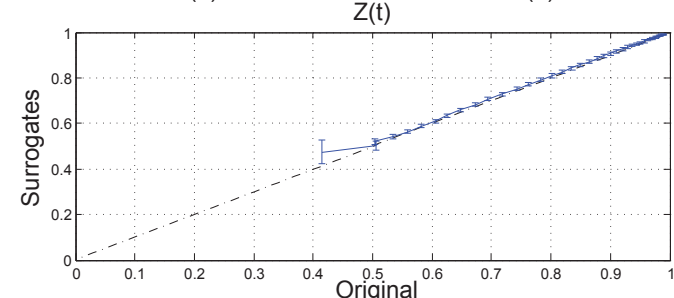

(c)

Fig. 2. Component-wise DVV scatter plots for pure quaternion signal $Q(t)$ (a) Henon map $X(t)$; (b) real world wind signal $Y(t)$; (c) linear AR(2) signal $Z(t)$.

Signal $X(t)$ was a realization of Henon map, a chaotic nonlinear signal, given by

$$
\begin{array}{r}
x_{n}=1-a x_{n-\beta}^{2}+y_{n-\beta} \\
y_{n}=b x_{n-\beta}
\end{array}
$$

where $\beta$ was set to unity, and parameters $a$ and $b$ were respectively set to 1.4 and 0.3 . The $\mathrm{x}$-component of the Henon map was used in simulations.

Signal $Y(t)$ was chosen as a vertical speed component of the real world wind signal, while $Z(t)$ was a benchmark linear $\mathrm{AR}(2)$ signal, given by

$$
z(k)=0.8 z(k-1)+0.1 z(k-2)+r(k)
$$

where $r(k)$ is white Gaussian noise signal of zero mean and unit variance.

Figure 2 shows the DVV scatter plots of the three components of $Q(t)$. Observe that for Henon map $X(t)$, the scatter plot deviates away from the bisector line, indicating its nonlinear behaviour. For the linear $\operatorname{AR}(2)$ signal $Z(t)$, the plot coincides with the bisector line, indicating its linear nature, whereas $Y(t)$ was either nonlinear or/and nonstationary.

In the modality analysis of quaternion IMFs, the three dimensional EMD algorithm was used to process the quaternion signal (5); the DVV method was then applied to all the three components of resulting quaternion IMFs, and the corresponding scatter plots were analysed. The metric in (4) was used to quantify the degree of similarity between the scatter plots of IMFs and those of the original signal shown in Figure 2. Figure 3 shows the DVV scatter plots of the components of the sum of 1 st and 2nd IMFs of a quaternion signal $Q(t)$ (Figure 3(a), 3(b), and 3(c)), and scatter plots of the remaining 

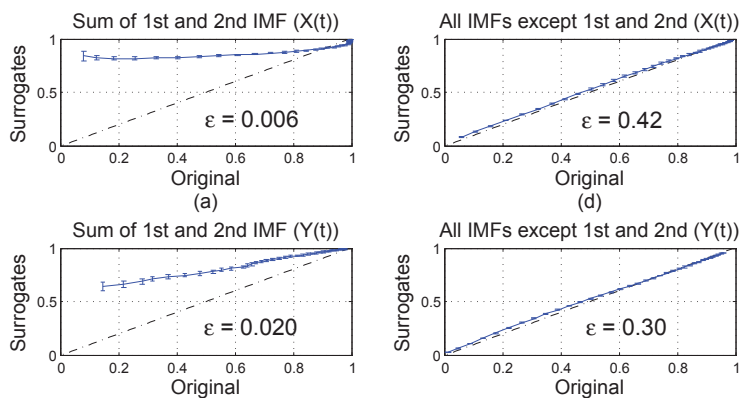

(b)

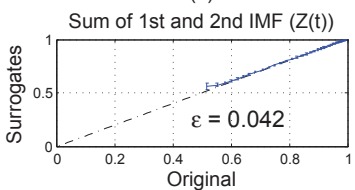

(c)

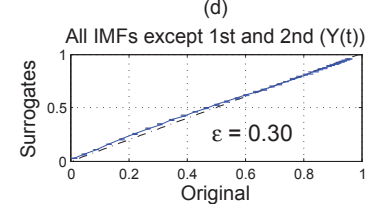

(e)

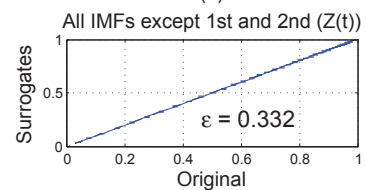

Fig. 3. DVV scatter plots and the corresponding values of similarity measure (4) for the three components of (a,b, and c) the sum of the 1st and 2nd IMFs generated from three dimensional EMD algorithm; (d,e, and f) the sum of remaining (all except IMF1 and IMF2) IMFs.

IMFs (Figure 3(d), 3(e), and 3(f)), together with their corresponding values of similarity metric. Notice that the sum of first two IMFs preserve the original nature for all three components of the input signal $Q(t)$, which is also manifested by the corresponding low values of similarity metric; while the sum of the rest of the IMFs have relatively large values of the similarity metric, showing that that the original signal modality is not contained in lower IMFs. In order to further explore the contribution of individual IMFs, the DVV scatter plots of the X-component of $Q(t)$ for the first four IMFs are separately plotted in Figure 4. Again, the values of similarity metric show that the first two IMFs preserve the signal modality, while the remaining IMFs are largely linear and predictable (deterministic).

\section{CONCLUSIONS}

We have presented a method for the modality analysis of quaternion IMFs generated from the three dimensional EMD algorithm. The Delay vector variance (DVV) method has been used to characterize the nature of a signal ("qualitative analysis"), whereby analysis of the IMFs corresponding to the original signal are characterised using a proposed similarity metric. The analysis has shown that the sum of first two IMFs is sufficient to preserve the signal modality for both the benchmark linear and nonlinear signals, and the real world wind data, whereas the sum of remaining IMFs exhibit largely linear and deterministic behaviour. This study will pave the way for applications of the three dimensional EMD method in conjunction with multivariate adaptive filtering algorithms and in data fusion.

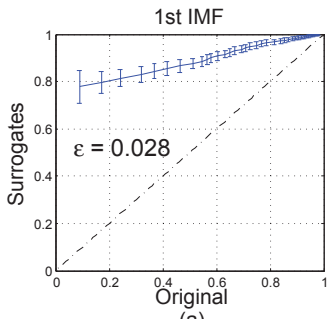

(a)

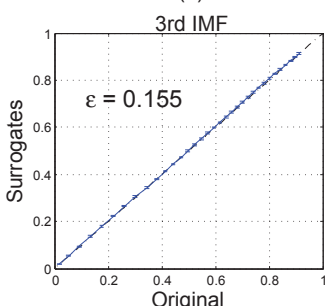

(c)

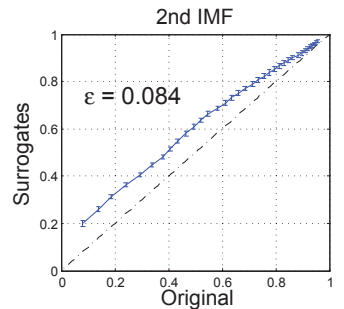

(b)

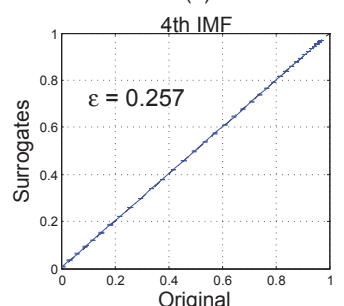

(d)
Fig. 4. DVV scatter plots of 1 st IMF (a); 2nd IMF (b); 3rd IMF (c); and 4th IMF (d) of $Y(t)$, generated by three dimensional EMD.

\section{REFERENCES}

[1] N. Huang, Z. Shen, S. Long, M. Wu, H. Shih, Q. Zheng, N. Yen, C. Tung, and H. Liu, "The empirical mode decomposition and Hilbert spectrum for non-linear and non-stationary time series analysis," Proceedings of the Royal Society of London, vol. 454, pp. 903-995, 1998.

[2] T. Tanaka and D.P. Mandic, "Complex empirical mode decomposition,” IEEE Signal Processing Letters, vol. 14, no. 2, pp. 101-104, 2006.

[3] M. U. Altaf, T. Gautama, T. Tanaka, and D.P. Mandic, "Rotation invariant complex empirical mode decomposition," in Proceedings of the International Conference on Acoustics, Speech, Signal Processing, 2007, pp. 1009-1112.

[4] G. Rilling, P. Flandrin, P. Goncalves, and J. M. Lilly, "Bivariate empirical mode decomposition," IEEE Signal Processing Letters, vol. 14, no. 12, pp. 936-939, 2007.

[5] N. Rehman and D. Mandic, "Three dimensional empirical mode decomposition," submitted to IEEE Transactions on Signal Processing, 2008.

[6] T. Gautama, D. Mandic, and M. M. Van Hulle, "The delay vector variance method for detecting determinism and nonlinearity in time series," Physica D, vol. 190, no. 3-4, pp. 167-176, 2004.

[7] D. Looney and D. Mandic, "A machine learning enhanced empirical mode decomposition," in Proceedings of the International Conference on Acoustics, Speech, Signal Processing, 2008, pp. 1897-1900.

[8] M. Chen, D. Mandic, P. Kidmose, and M. Ungstrup, "Qualitative assessment of intrinsic mode functions of empirical mode decomposition," in Proceedings of the International Conference on Acoustics, Speech, Signal Processing, 2008, pp. 1905-1908.

[9] D. P Mandic et al., Signal Processing Techniques for Knowledge Extraction and Information Fusion, Springer, 2008. 